\title{
Article \\ Design of Power Splitters Based on Hybrid Plasmonic Waveguides
}

\author{
Xiaoyan Shi ${ }^{1}$, Wu Yang ${ }^{1, *}$, Huaizhong Xing ${ }^{2, *}$ and Xiaoshuang Chen ${ }^{3}$ \\ 1 College of Science, Henan University of Technology, Zhengzhou 450001, China; siyer@haut.edu.cn \\ 2 Department of Applied Physics and State Key Laboratory for Modification of Chemical Fibers and Polymer \\ Materials, Donghua University, Songjiang District, Shanghai 201620, China \\ 3 National Laboratory for Infrared Physics, Shanghai Institute of Technical Physics, Chinese Academy of \\ Sciences, 500 Yutian Road, Shanghai 200083, China; xschen@mail.sitp.ac.cn \\ * Correspondence: yang5@haut.edu.cn (W.Y.); xinghz@dhu.edu.cn (H.X.)
}

check for updates

Citation: Shi, X.; Yang, W.; Xing, H.; Chen, $X$. Design of Power Splitters Based on Hybrid Plasmonic

Waveguides. Appl. Sci. 2021, 11, 8644. https://doi.org/10.3390/app11188644

Academic Editor: Dror Malka

Received: 6 August 2021

Accepted: 15 September 2021

Published: 17 September 2021

Publisher's Note: MDPI stays neutral with regard to jurisdictional claims in published maps and institutional affiliations.

Copyright: (C) 2021 by the authors. Licensee MDPI, Basel, Switzerland. This article is an open access article distributed under the terms and conditions of the Creative Commons Attribution (CC BY) license (https:// creativecommons.org/licenses/by/ $4.0 /)$.

\begin{abstract}
Plasmonic power splitters based on hybrid plasmonic waveguides (HPWs) are proposed and investigated. The HPW consists of a high-permittivity semiconductor nanowire embedded in a $\mathrm{SiO}_{2}$ dielectric film near a metal surface. The propagation behaviors of Surface Plasmon Polaritons (SPPs) in HPWs are numerically simulated by the 3D finite-difference time-domain (FDTD) method. The incident field is transferred from the middle waveguide to the waveguides on both sides due to the coupling between adjacent waveguides. The intensity distributions can be explained by the multimode interference of SPPs supermodes. According to the field intensity distribution of five HPWs, we design a $1 \times 3$ power splitter and a $1 \times 2$ power splitter by reducing the length of some specific waveguides.
\end{abstract}

Keywords: power splitters; surface plasmon polaritons; hybrid plasmonic waveguides

\section{Introduction}

Surface Plasmon Polariton (SPP) waveguides can break the diffraction limitation and are regarded as the best candidate to design miniaturized photonic integrated circuits [1,2] All kinds of plasmonic waveguides have been designed, such as the dielectric loaded metal, metal wedges, slot and gap waveguides, and hybrid plasmonic waveguides [3,4]. Among these plasmonic waveguides, the hybrid plasmonic waveguide (HPW) is one of the most promising alternatives for on-chip nanophotonics due to the ability of subwavelength confinement and long propagation lengths [3].

In photonic integrated circuits, a power splitter is an essential component, which can distribute the energy of incident light to different propagation directions. Various plasmonic power splitters have been proposed, such as $1 \times 2$ splitters [5-8], $1 \times 3$ splitters [9], $1 \times \mathrm{N}$ splitters [10-12], and variable transmission splitters [13-15]. The overwhelming majority of these plasmonic splitters are designed on the basis of metal-insulator-metal (MIM) waveguides, which are composed of two metallic layers and a dielectric layer [5-15]. However, there are few researches on the power splitter based on HPWs. The main reason is that an MIM waveguide could be simplified to a two-dimensional structure and a HPW cannot be simplified to a two-dimensional structure, of which the computational complexity of a 3D structure is much larger than that of a 2D structure. Due to the excellent performance of the HPWs, it is necessary to design a 3D power splitter based on HPWs for the next generation of integrated photonic circuits.

In this paper, we present several new power splitters based on hybrid plasmonic waveguides. The propagation behaviors of Surface Plasmon Polaritons (SPPs) in HPWs are numerically simulated by the 3D finite-difference time-domain (FDTD) method. According to the field distribution of five HPWs, we reduce the length of some specific waveguides and design different types of power splitters. The power splitters based on HPWs are 
fully compatible with semiconductor fabrication techniques and can be widely used in integrated photonics structures.

\section{Materials and Methods}

The hybrid mode in the single HPW was discussed in detail in ref. [3]. Here, we considered a coupled system composed of five HPWs, shown in Figure 1. The HPW consists of a high-permittivity semiconductor nanowire embedded in a low-permittivity dielectric near a metal surface [3]. The semiconductor nanowire was GaAs, of which the relative permittivity is 12.25 . The low-permittivity dielectric was $\mathrm{SiO}_{2}$, with relative permittivity 2.25. The relative permittivity of Ag was taken to be the measured value of $\varepsilon=-129+3.3 j$ at the telecommunications wavelength $\lambda=1550 \mathrm{~nm}$ [16]. The diameter of the GaAs nanowire was $\mathrm{d}=200 \mathrm{~nm}$ and the dielectric gap was $\mathrm{h}=50 \mathrm{~nm}$. The distance between adjacent waveguides was $S=500 \mathrm{~nm}$. The plasmonic mode supported by a single HPW is shown in Figure 1c, which was consistent with the results of ref. [3].

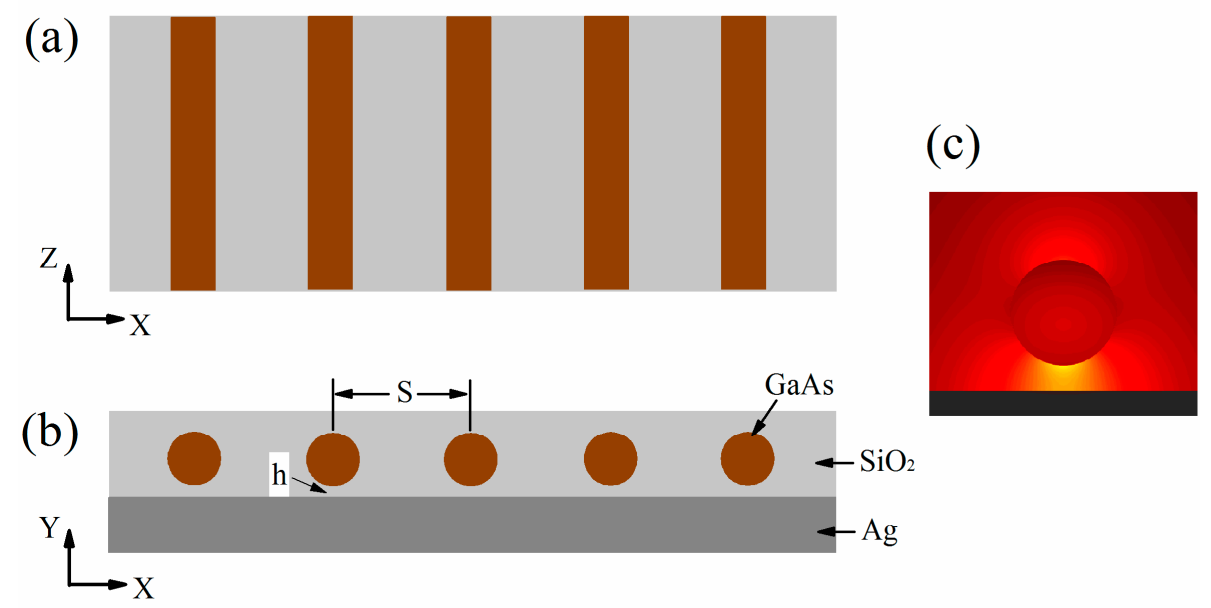

Figure 1. Schemes of five adjacent HPWs. (a) The top view of the HPWs. (b) The front view of the HPWs. The HPWs consist of GaAs nanowires embedded in $\mathrm{SiO}_{2}$ dielectric. The diameter of the GaAs nanowire was $200 \mathrm{~nm}$ and the gap was $\mathrm{h}=50 \mathrm{~nm}$. The distance between adjacent waveguides was $\mathrm{S}=500 \mathrm{~nm}$. (c) The field distribution of the plasmonic mode for the single HPW.

We numerically simulated the propagation behavior of SPPs in five HPWs by the 3D FDTD method. The grid size was $\mathrm{dx}=\mathrm{dz}=10 \mathrm{~nm}$ and the time increment was $\Delta \mathrm{t}=\Delta \mathrm{s} / 2 \mathrm{c}$. A perfectly matching layer was adopted to absorb the outgoing electromagnetic wave around the computational domain.

\section{Results and Discussion}

The plasmonic mode was excited by a Gaussian beam, which is normally focused onto the end of the middle waveguide [17]. The arrangement of the five HPWsis shown in Figure 2a. The intensity distributions in the XZ plane are shown in Figure 2b, where the pattern was very complex due to multimode interference effects [18]. In ref. [18], the principles and properties of multimode interference devices are presented for conventional dielectric waveguides. To the device of five HPWs, supermodes were formed due to the coupling between adjacent waveguides $[19,20]$. Customarily, supermodes are the modes of propagation of a periodic array of waveguides, which consists of $N$ identical waveguides [20]. 


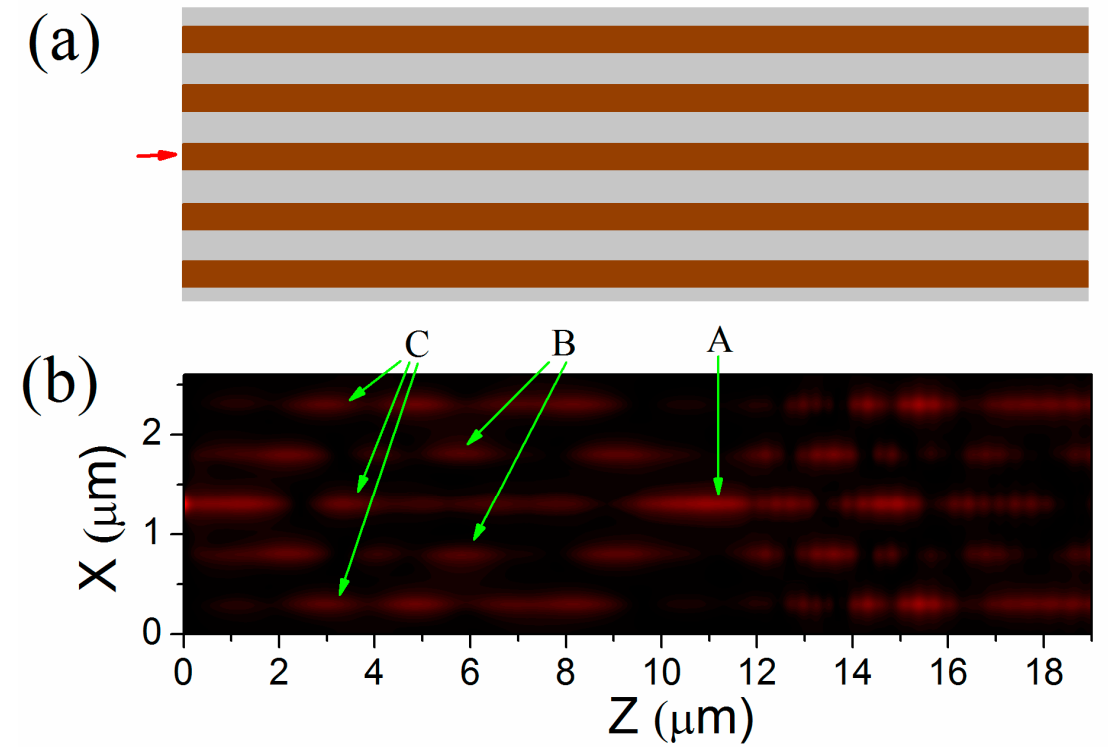

Figure 2. (a) The arrangement of five HPWs in xz plane. (b) The propagation behavior of SPPs in five HPWs.

The wave functions of the supermodes were written as linear combinations of the unperturbed wave functions of the individual waveguides:

$$
H_{S}(x, z)=\sum_{n=1}^{N} C_{s, n} \psi_{n}(x, z)
$$

The total field in a periodic array of waveguides can be expressed as a sum of all supermodes, which can be written as:

$$
\varphi(x, z)=\sum_{s=1}^{N} a_{s} H_{s}(x) e^{-i \beta_{s} z}
$$

$\beta_{S}$ is the propagation constant of a single supermode, $a_{s}$ is the weight coefficient of excited supermodes [19].

Due to the incident field of SPPs being the input from the middle waveguide, even supermodes can be excited. These excited supermodes produced a multimode interference and formed a self-imaging phenomenon. The corresponding $N$-fold images were expected to form at distances [18]:

$$
L=\frac{P}{N}\left(\frac{3 L_{\pi}}{4}\right)(P=1,2,3, \ldots)
$$

where $P$ denotes the periodic nature of the imaging along the waveguides, $L_{\pi}=\pi /\left(\beta_{1}-\beta_{2}\right)$ is the beating length of the two lowest-order supermodes, and $\beta_{1}$ and $\beta_{2}$ are propagation constants of the two supermodes. In Figure $2 b$, the arrow A shows the position of a one-fold image $(N=1)$, where $L_{A}=11.2 \mu \mathrm{m}$. According to Equation (3), the positions of two-fold images and three-fold images were at $L_{A} / 2$ and $L_{A} / 3$, which are shown by the arrows $B$ and $\mathrm{C}$.

The coupling transmission of the cross section between adjacent waveguides is shown in Figure 3, which corresponded to different $\mathrm{z}$ coordinates. It can be seen from Figure 3 that the energy was transferred from the middle waveguide to the waveguides on both sides due to the coupling between adjacent waveguides. 
(a)

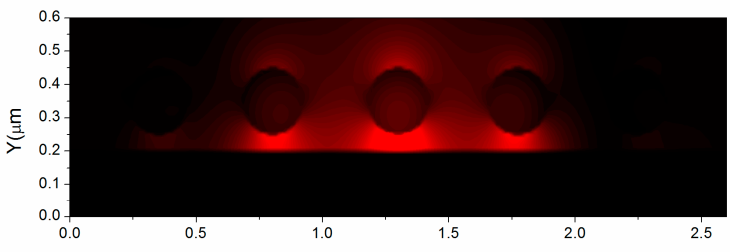

(b)

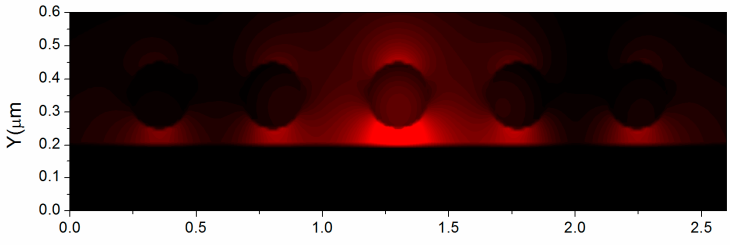

(c)

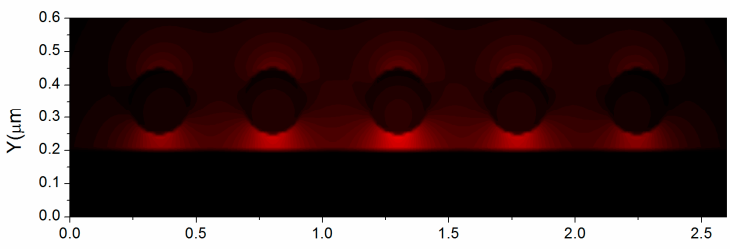

(d)

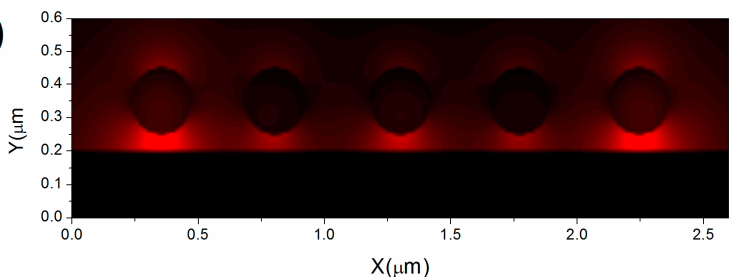

Figure 3. The cross section with different $\mathrm{z}$ coordinates. (a) $\mathrm{z}=1.8 \mu \mathrm{m} ;(\mathbf{b}) \mathrm{z}=4.0 \mu \mathrm{m} ;(\mathbf{c}) \mathrm{z}=4.1 \mu \mathrm{m}$; (d) $\mathrm{z}=4.2 \mu \mathrm{m}$.

As the distance increased, the coupling between waveguides became weaker. Figure 4 shows the propagation behavior of SPPs in three HPWs, in which the distance between waveguides was doubled. We removed the 2 nd and the 4 th waveguides on the basis of Figure 3. As can be seen from Figure 4, the length required for energy coupling from one waveguide to adjacent waveguides became longer. When the length of the HPWs was less than $10 \mathrm{um}$, the coupling between waveguides could be ignored. This also illustrates that the 5 th waveguide had little effect on the 3rd waveguide in Figure 2.

(a)

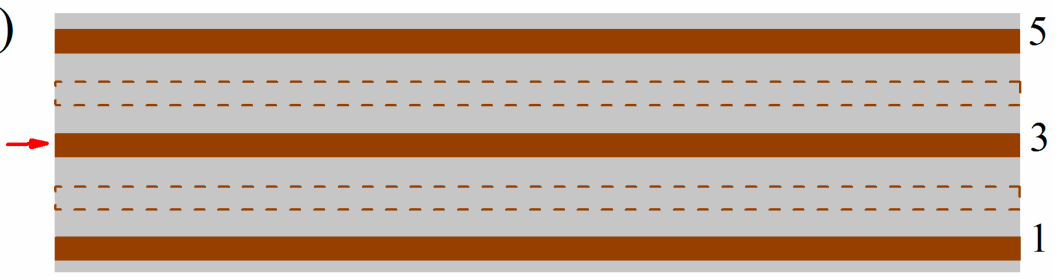

(b)

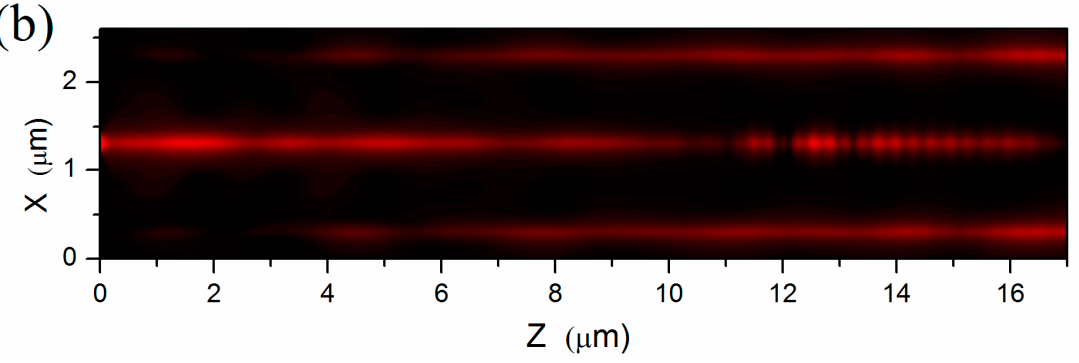

Figure 4. (a) The arrangement of three HPWs in $x z$ plane. We removed the 2 nd and the 4 th waveguides on the basis of Figure 3 and the distance between adjacent waveguides was $1000 \mathrm{~nm}$. (b) The propagation behavior of SPPs in three HPWs. 
Based on the simulation results of Figure $2 b$, we could design a $1 \times 3$ power splitter by the self-imaging phenomenon [18]. In Figure $2 b$, the positions of 3 -fold images were at $L_{A} / 3$, as shown by the arrows $C$. When $z=3.2 \mu \mathrm{m}$, the field intensity of the $2 \mathrm{nd}$ and the 4th waveguides was zero, in which the field energy was coupled to the $3 \mathrm{rd}$ and the 5 th waveguides. If we reduced the length of the 2 nd and the 4 th waveguides to $3.2 \mu \mathrm{m}$, a $1 \times 3$ power splitter could be obtained. The scheme is shown in Figure 5a. The intensity distribution is shown in Figure 5b. The field distribution is shown in Figure 5c. It can be seen that the incident field was transmitted into the 1st, $3 \mathrm{rd}$, and 5 th waveguides. The ratio of the output power was about 1:1:1. The transmission efficiency, $\eta=P_{\text {out }} / P_{\text {in }}$, was $91 \%$, where $P_{\text {out }}$ was the power in the output and $P_{\text {in }}$ was the power in the input. The output power $P_{\text {out }}$ was the sum of the 1 st, 3 rd, and 5 th waveguides. The energy loss mainly came from two aspects. One was due to the reflection of the waveguide port. When the input field was transmitted in the 2nd and 4th waveguides, part of the energy was reflected by the waveguide ports. The other aspect was the ohmic loss of the waveguide itself [3].
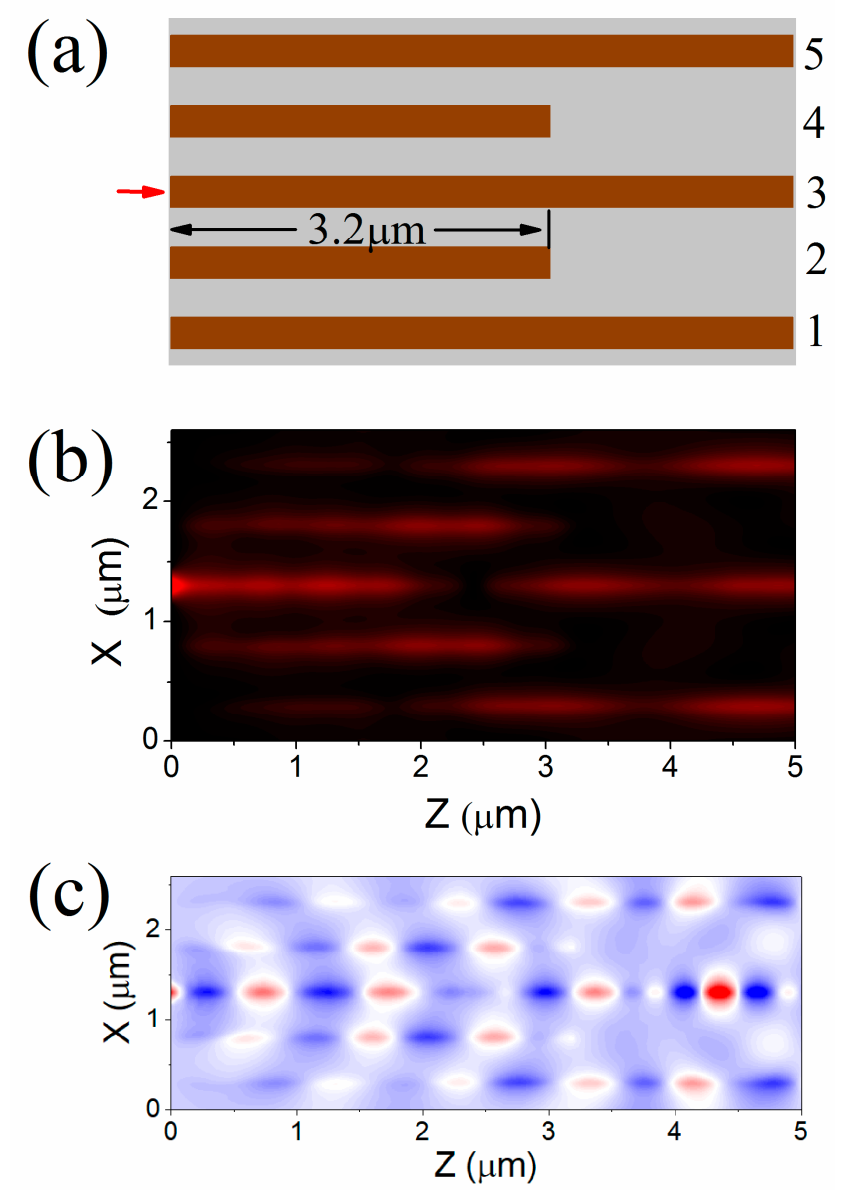

Figure 5. (a) Schemes of $1 \times 3$ power splitter based on five HPWs. (b) The intensity distribution of SPPs in the power splitter. (c) The field distribution of SPPs in the power splitter.

We could also design a $1 \times 2$ power splitter according to the field intensity distribution of Figure $5 b$. We reduced the length of the 3rd waveguide to $2.6 \mu \mathrm{m}$, as shown in Figure 6a. The field intensity distribution is shown in Figure $6 \mathrm{~b}$. The incident field was transmitted into the 1st and 5th waveguides. The ratio of the output power was 1:1. The output power was the sum of the 1 st and 5 th waveguides. The transmission efficiency $\eta$ was $77 \%$. 

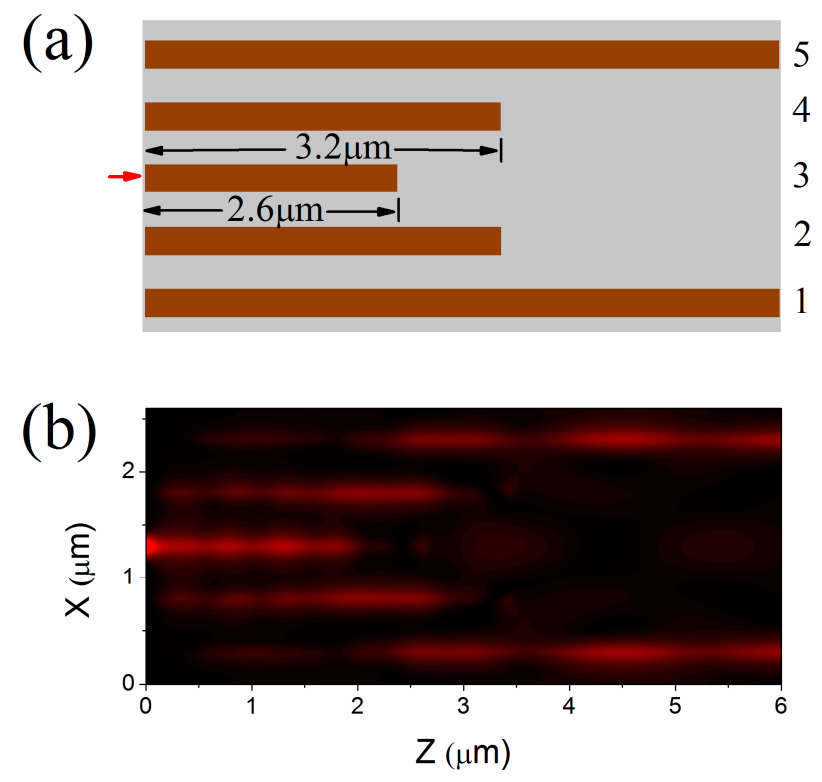

Figure 6. (a) Schemes of $1 \times 2$ power splitter based on five HPWs. (b) The intensity distribution of SPPs in the power splitter.

Another scheme for $1 \times 2$ power splitter could be designed by three HPWs, as shown in Figure 7a. The length of the middle waveguide was $2.6 \mu \mathrm{m}$. The field intensity distribution is shown in Figure $7 \mathrm{~b}$. The incident field was coupled to the adjacent waveguides. The ratio of the output power was also 1:1. The transmission efficiency $\eta$ was $92 \%$, which was more efficient than the scheme of Figure 6. The principal reason was that the number of the middle waveguides in Figure 6 was three and the number in Figure 7 was one, in which the input energy was reflected by the output ends of the middle waveguides.

(a)
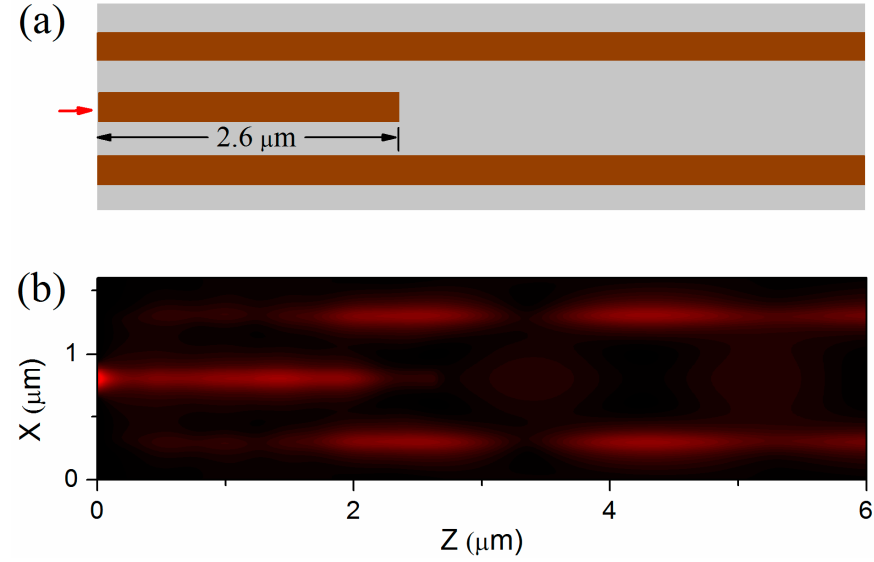

Figure 7. (a) Schemes of $1 \times 2$ power splitter based on three HPWs. (b) The intensity distribution of SPPs in the power splitter.

We compared the power splitters proposed in this work with other power splitters previously published, which are shown in Table 1 . The previous power splitters were mainly based on 2D MIM waveguides. Here, the power splitter was based on 3D HPWs, which was more suitable for integrated photonics structures and had a higher transmission efficiency. 
Table 1. The comparison of various power splitters.

\begin{tabular}{ccc}
\hline Power Splitter & Wavelength & Transmission Efficiency \\
\hline Ref. [5] (2D MIM) & $600 \mathrm{~nm}-900 \mathrm{~nm}$ & $50-80 \%$ \\
Ref. [6] (2D MIM) & $1550 \mathrm{~nm}$ & $80-90 \%$ \\
Ref. [9] (2D MIM) & $1550 \mathrm{~nm}$ & $80-88 \%$ \\
Ref. [14] (2D MIM) & $1550 \mathrm{~nm}$ & $80-90 \%$ \\
Ref. [15] (2D MIM) & $537 \mathrm{~nm}$ & $76.3 \%$ \\
In this work (3D HPWs) & $1550 \mathrm{~nm}$ & $77-92 \%$ \\
\hline
\end{tabular}

\section{Conclusions}

We presented several new power splitters based on five HPWs. The propagation behaviors of SPPs in five HPWs were numerically simulated by the 3D FDTD method. The incident field was transferred from the middle waveguide to the waveguides on both sides due to the coupling between adjacent waveguides. The intensity distributions could be explained by the multimode interference of SPPs supermodes. According to the field intensity distribution of five HPWs, we reduced the length of some specific waveguides and designed a $1 \times 3$ power splitter and a $1 \times 2$ power splitter, which the ratios of the output power being 1:1:1 and 1:1, respectively.

Author Contributions: Conceptualization, X.S. and W.Y.; methodology, H.X.; software, X.C.; validation, W.Y., H.X. and X.C.; formal analysis, X.S.; investigation, X.S.; resources, H.X.; data curation, X.C.; writing—original draft preparation, X.S.; writing—-review and editing, W.Y.; visualization, W.Y.; supervision, H.X.; project administration, X.C.; funding acquisition, X.S. All authors have read and agreed to the published version of the manuscript.

Funding: This research was supported by the Doctoral Foundation of Henan University of Technology (2016BS010), the Fundamental Research Funds for Henan Provincial Colleges and Universities in Henan University of Technology (2016QNJH13), the Key Scientific and Technological Research Projects in Henan Province (192102210077), and the Natural Science Foundation of Shanghai (21ZR1402200).

Institutional Review Board Statement: Not applicable.

Data Availability Statement: The data presented in this study are available on request from the corresponding author.

Conflicts of Interest: The authors declare no conflict of interest.

\section{References}

1. Ozbay, E. Plasmonics: Merging Photonics and Electronics at Nanoscale Dimensions. Science 2006, 311, 189-193. [CrossRef] [PubMed]

2. Zia, R.; Schuller, J.A.; Chandran, A.; Brongersma, M.L. Plasmonics: The next chip-scale technology. Mater. Today 2006, 9, 20-27. [CrossRef]

3. Oulton, R.F.; Sorger, V.J.; Genov, D.A.; Pile, D.F.P.; Zhang, X. A hybrid plasmonic waveguide for subwavelength confinement and long-range propagation. Nat. Photonics 2008, 2, 496-500. [CrossRef]

4. Kinsey, N.; Ferrera, M.; Shalaev, V.M.; Boltasseva, A. Examining nanophotonics for integrated hybrid systems: A review of plasmonic interconnects and modulators using traditional and alternative materials. JOSA B 2015, 32, 121-142. [CrossRef]

5. Wen, K.; Hu, Y.; Chen, L.; Zhou, J.; Lei, L.; Guo, Z. Design of an optical power and wavelength splitter based on subwavelength waveguides. Lightwave Technol. 2014, 32, 3020-3026. [CrossRef]

6. Butt, M.A.; Khonina, S.N.; Kazanskiy, N.L. Ultra-short lossless plasmonic power splitter design based on metal-insulator-metal waveguide. Laser Phys. 2019, 30, 016201. [CrossRef]

7. Ghasemi, M.R.; Bayati, M.S. Proposal for metal-insulator-metal plasmonic power splitter and demultiplexer suitable for implementation in optical switches. IET Optoelectron. 2021, 15, 200-206. [CrossRef]

8. Wahsheh, R.A. Ultra-compact broadband 3-db metal-dielectric-metal plasmonic power splitter. J. Mod. Opt. 2021, 68, 153-160. [CrossRef]

9. Nozhat, N.; Granpayeh, N. Analysis of the plasmonic power splitter and MUX/DEMUX suitable for photonic integrated circuits. Opt. Commun. 2011, 284, 3449-3455. [CrossRef] 
10. Chen, C.H.; Liao, K.S. 1xN plasmonic power splitters based on metal-insulator-metal waveguides. Opt. Express 2013, 21, 4036-4043. [CrossRef] [PubMed]

11. Ayad, M.A.; Obayya, S.S.A.; Swillam, M.A. Submicron 1xN ultra wideband MIM plasmonic power splitters. Lightwave Technol. 2014, 32, 1814-1820. [CrossRef]

12. Ayad, M.A.; Swillam, M.A. Mid-infrared plasmonic power splitters. IEEE Photonics Technol. Lett. 2016, 28, 2431-2434. [CrossRef]

13. Chang, Y.; Chen, C.-H. Design of a broadband plasmonic unequal-power splitter with a rectangular ring resonator. Plasmonics 2015, 10, 739-743. [CrossRef]

14. Guo, Y.; Yan, L.; Pan, W.; Luo, B.; Wen, K.; Guo, Z.; Li, H.; Luo, X. A plasmonic splitter based on slot cavity. Opt. Express 2011, 19, 13831-13838. [CrossRef] [PubMed]

15. Ye, Y.; Xie, Y.; Song, T.; Wang, Y.; Chai, J.; Liu, B.; Liu, Y. Design of a Novel Plasmonic Splitter with Variable Transmissions and Selectable Channels. IEEE Trans. Nanotechnol. 2019, 18, 617-625. [CrossRef]

16. Palik, E.D. Handbook of Optical Constants of Solids; Academic Press: New York, NY, USA, 1985.

17. Bian, Y.; Gong, Q. Metallic-nanowire-loaded silicon-on-insulator structures: A route to low-loss plasmon waveguiding on the nanoscale. Nanoscale 2015, 7, 4415-4422. [CrossRef] [PubMed]

18. Soldano, L.B.; Pennings, E.C.M. Optical Multi-Mode Interference Devices Based on Self-Imaging: Principles and Applications. Lightwave Technol. 1995, 13, 615-627. [CrossRef]

19. Shi, X.; Yang, W.; Xing, H.; Chen, X. Discrete plasmonic Talbot effect in finite metal waveguide arrays. Opt. Lett. 2015, 40, 1635-1638. [CrossRef] [PubMed]

20. Yariv, A.; Yeh, P. Photonics: Optical Electronics in Modern Communications; Oxford University Press: New York, NY, USA, 2007. 